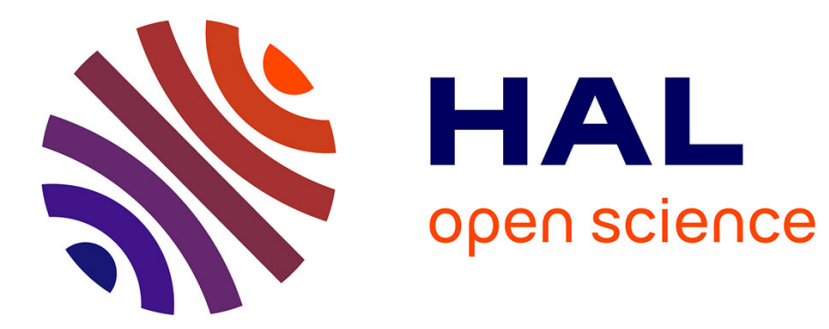

\title{
OPTICAL IMAGING AND CHEMOTHERAPY
}

Guillaume Bort, Simona Mura, Patrick Couvreur

\section{To cite this version:}

Guillaume Bort, Simona Mura, Patrick Couvreur. OPTICAL IMAGING AND CHEMOTHERAPY. Nanotheranostics for Personalized Medicine, WORLD SCIENTIFIC, pp.83-114, 2016, 10.1142/9789814713535_0005. hal-02784812

\section{HAL Id: hal-02784812 \\ https://hal.science/hal-02784812}

Submitted on 10 Nov 2020

HAL is a multi-disciplinary open access archive for the deposit and dissemination of scientific research documents, whether they are published or not. The documents may come from teaching and research institutions in France or abroad, or from public or private research centers.
L'archive ouverte pluridisciplinaire HAL, est destinée au dépôt et à la diffusion de documents scientifiques de niveau recherche, publiés ou non, émanant des établissements d'enseignement et de recherche français ou étrangers, des laboratoires publics ou privés. 


\section{OPTICAL IMAGING AND CHEMOTHERAPY}

Guillaume Bort, Simona Mura, Patrick Couvreur

Université Paris-Sud, Faculté de Pharmacie, 5 rue

Jean-Baptiste Clément, 92296 Châtenay-Malabry

cedex, France.

CNRS UMR 8612, Institut Galien Paris-Sud, 5 rue

Jean-Baptiste Clément, 92296 Châtenay-Malabry

cedex, France.

E-mail: patrick.couvreur@u-psud.fr 


\begin{abstract}
Nanoscale drug delivery systems which combine in a single tool imaging and therapeutic functions, (i.e., nanotheranostics) have attracted a great deal of interest due to the possibility to monitor in real-time both distribution/drug release and the effectiveness of the treatment thus, allowing an immediate adjustment of the therapeutic protocol. Among the various modalities, this chapter will focus on the optical imaging combined to the delivery of anticancer drugs and the main achievement obtained in this field will be discussed. Nanotheranostics displaying multi-imaging properties will be also presented.
\end{abstract}




\section{Table of Contents}

1. Introduction

2. Fluorescent drug containing nanotheranostics

3. Dye containing biodegradable polymeric nanotheranostics

4. Mesoporous silica-based nanotheranostics

5. Semiconductor quantum dots-based nanotheranostics

6. Lanthanide-doped upconversion nanotheranostics

7. Gold containing nanotheranostics

8. Iron oxide-based nanotheranostics

9. Radioluminescent nanotheranostics

10. Miscellaneous

11. Conclusion 


\section{Introduction}

A great deal of attention is currently focused on the emerging field of "personalized medicine", which holds the promises of enabling $(i)$ detection and monitoring over time of an individual patient's disease and (ii) on-demand tuning of the therapeutic treatments. In this context, a large variety of theranostic nanosystems ("nanotheranostics"), which integrate therapeutic and imaging functions in the same tool, have been designed in the last decade to achieve a better understanding on the mechanism and efficiency of drug release as well as for monitoring in real-time the treatment efficacy. ${ }^{1}$ Among the numerous approaches, nanotheranostics which combine therapeutic molecules and molecules or particles detectable by optical imaging aroused high interest and have been widely explored. Indeed, optical imaging is one of the most promising imaging modality due to its high sensitivity, high spatiotemporal resolution for both in vivo and in vitro imaging, and relatively low cost. Investigation of processes at the cellular and molecular levels is also possible. Many theranostic systems require the use of ultraviolet (UV) excitation to be activated or detected. However, while UV light showed great possibility for controlling/monitoring systems in vitro, the in vivo application is severely limited by the low penetration depth (due to strong light absorption and scattering of soft tissues) and induction of high cellular damage. On the other hand, near-infrared (NIR) light (700-900 nm) exhibits low tissue absorbance, leading to deeper penetration in living tissues $(3 \mathrm{~cm}$ in thick animal tissues and up to $10 \mathrm{~cm}$ in less attenuating organs such as breast $)^{2,3}$ and displays lower damaging potential. For this reason the development of activating/monitoring nanotheranostics based on NIR light is more attractive. Metal containing probes, such as semiconductor quantum dots (QD), lanthanides (Ln) or gold, are playing a major role in this field. ${ }^{4-6}$

While the first developed systems were used only to confirm the presence of the drug carrier at the targeted site, further sophistication of the technology enabled to monitor and quantify the in situ drug release. Finally, recently designed stimuli-sensitive nanotheranostics offer a way 
for a precise "on-demand" control of the drug release. In this chapter, examples of the most important achievements realized with nanotheranostics combining non-invasive optical imaging and cancer chemotherapy are discussed.

\section{Fluorescent drug-containing nanotheranostics}

Fluorescent drugs enable easy tracking of drug-loaded nanocarriers without addition of any complementary fluorescent tag. Doxorubicin (Dox), an anthracycline antibiotic inducing cell death mainly by intercalating DNA, ${ }^{7}$ is the most commonly used anticancer drug displaying fluorescent properties. Due to the auto-quenching of Dox fluorescence, the drug release from a carrier can be detected by monitoring the variation of the fluorescence signal over time. Such shift can also confirm the effectiveness of a specific stimulus to trigger the drug release. For instance, the strong increase of the fluorescent signal observed after exposure of poly(ethylene glycol)- $b$-poly( $\gamma$-benzyl L-glutamate) (PEG-SS-PBLG) micelles, bearing dox through a disulfide bond, to glutathione (GSH) concentration higher than $1 \mathrm{mM}$, confirmed the bioreductible character of the system and the possibility to trigger Dox release. ${ }^{8}$

However, although easy to detect, several parameters have to be taken into account when the fluorescence signal is used to quantify the Dox. Indeed, the Dox concentration itself, the local microenvironment and the interaction with cellular components may influence Dox fluorescence intensity by changing its quantum efficiency. For instance, Dox fluorescence is dramatically quenched upon intercalation between the DNA base pairs, thus measured signal intensity do not represent the Dox concentration and might not correlate with the drug therapeutic efficacy. ${ }^{9,10}$ Nevertheless, precise Dox quantification in tumor tissue could be achieved by application of Monte Carlo-based models of light-tissue interaction ${ }^{11}$ or other approaches such as the forward-adjoint fluorescence model. ${ }^{12}$ Besides Dox, mitoxantrone ${ }^{10}$ and camptothecin $(\mathrm{Cpt})^{13}$ could also be detected due to their intrinsic fluorescence. 


\section{Fluorescently labelled nanotheranostics}

In the absence of fluorescent drug molecules, nanocarrier distribution and/or drug release can be monitored by designing fluorescently-tagged systems. Various NIR dyes such as indocyanine green (ICG), Cyanine5.5 (Cy5.5), lipophilic 3, 3'-dioctadecyloxacarbocyanine perchlorate $(\mathrm{DiO})$ are commonly employed. The dye can be either physically encapsulated or covalently linked to the building material. In both case, quantification of dye loading is required for complete and reliable characterization of the system. When biodegradable polymers are used, low-destructive methods such as combination of spectroscopic and microscopic techniques (e.g., steady-state fluorescence, anisotropy, and emission and anisotropy lifetime decay profiles) are recommended to avoid inaccurate dye loading assessment due to the degradation of the polymer itself. ${ }^{14}$

Physical encapsulation of the amphipilic ICG into cytochrome $c$-loaded nanoparticles (NPs) made of a water-soluble hyperbranched polyhydroxyl polymer was proposed to track them in vitro and easily quantify their uptake by MCF7 and A549 cells by NIR fluorescence (NIRF). In addition, the dye enabled to assess the role of receptor-mediated internalization when these NPs were functionalized with folic acid (FA) ${ }^{15}$ Co-loading of Cy5.5 and paclitaxel (Ptx) into chitosan-based NPs, known for their stability in serum, deformability (compared to the polystyrene beads) and rapid uptake in tumor cells was also reported. ${ }^{16}$ In vivo whole-body NIRF imaging in early-stage SCC7 (murine squamous cell carcinoma cells) tumor-bearing mice showed a higher tumor accumulation of these NPs compared to rigid polystyrene beads. Fluorescence intensity was proportional to NP accumulation and tumor size, thus the kinetic of dye accumulation enabled to monitor the therapeutic response and optimise the treatment protocol. Highest tumor accumulation was achieved between 1 and 3 days post-injection and 
persisted for up to 10 days while a considerably low uptake was observed in the liver and spleen. The optimized therapeutic protocol derived from NIRF imaging data (20 mg Ptx/kg body weight, administered using 4 injections every 3 days) led to efficient antitumor activity significantly enhancing animal survival and minimizing the severe toxicity associated with free Ptx. Encapsulation of hydrophobic dyes and drug molecules into aminoacid-based NPs was also reported. ${ }^{17}, 18$

Formulation of nanotheranostics using chemically modified materials was chosen by other groups in order to avoid any possible misinterpretation of the imaging results as consequence of fluorescent dye desorption and/or release. For example, dye-labelled lipids were introduced in the liposomal formulation when fluorescent RGD-functionalized Dox-loaded liposomes were prepared by the thin film hydration method, mixing DSPC, cholesterol, DOPE, DSPEmPEG2000, DSPE-cRGDfK and BODIPY-modified DOPE. Compared to the free drug (1 mg. $\mathrm{Kg}^{-1}$, intravenously injected), these liposomes showed 15 -fold increase in antimetastatic activity in clinically relevant pancreatic (murine R40P) and renal (human SN12C-RFP) cell orthotopic models of spontaneous metastasis, whereas no weight loss was observed. ${ }^{19}$ The presence of BODIPY-DOPE in the liposome membrane allowed in vivo tracking and observation by intravital microscopy of liposomes binding to the tumor neovasculature. Similarly, fluorescent-labelled Dox-loaded liposomes functionalised with a tumor cells-binding peptide (CSNIDARAC) were easily prepared by inserting the Cy7.5-modified-DOPE in the lipid bilayer. ${ }^{20}$ Significant tumor accumulation was observed by non-invasive in vivo imaging, $2 \mathrm{~h}$ after intravenous injection of the functionalised liposomes in $\mathrm{H} 460$ lung tumor-bearing mice ( $2 \mathrm{mg}$ Dox/kg body weight). The higher tumor accumulation of these liposomes compared to the non-functionalized counterpart resulted in a higher inhibition of tumor growth. 
With the aim to combine imaging with a spatiotemporal controlled delivery of drugs, stimuliresponsive nanotheranostics were also designed. $\mathrm{pH}$-sensitive sugar-functionalised Dox-loaded fluorescent micelles were prepared blending poly( $N$-isopropyl acrylamide-co-methacryl acid)$g$-poly(D,L-lactide), mPEG-PLA, galactosamine-PEG-PLA and FITC-PEG-PLA. ${ }^{21} \mathrm{~A}$ significant release of Dox (70\% in $140 \mathrm{~h}$ ) was measured in PBS at pH 5.0, leading to dramatic cytotoxicity on HepG2 human hepatocellular carcinoma cells. Dox and FITC fluorescent signals were used to monitor, by confocal laser scanning microscopy (CLSM), intracellular distribution in HeLa cells of both micelles and released drug. In another study, both empty and Dox-loaded NPs were prepared with Cy5.5-labeled glycol chitosan modified with different degrees of hydrophobic $N$-acetyl histidine, which endowed the NPs with pH-responsiveness. ${ }^{22}$ Non-invasive in vivo imaging was used to monitor NP biodistribution and tumor targeting efficiency which was strongly influenced by the presence of $N$-acetyl histidine and Dox in the NP. Indeed, Dox loading showed to affect the physicochemical properties of the chitosan NPs resulting in different biodistribution profiles between empty and Dox-loaded NPs due to the stabilisation effect of the Dox in the core of the chitosan NPs provided by hydrophobic interactions. A higher tumor accumulation was observed with empty NPs containing the highest amount of $N$-acetyl histidine (7.8 wt\%), whereas for Dox-loaded ones the highest accumulation was reached in presence of only $3.3 \mathrm{wt} \%$ of $N$-acetyl histidine. Interestingly similar biodistribution profiles were obtained with both optical and scintigraphic ( ${ }^{131}$ I-labelled NPs) imaging. The most important targeting ability was then associated with a significant anticancer efficacy in HT29 tumor bearing mice.

Examples of responsive nanocarriers in which stimuli tuned both drug release and fluorescence emission were also reported. For instance, Satchi-Fainaro et al. conjugated both Cy5 and Ptx to $N$-(2-hydroxypropyl) methacrylamide (HMPA) copolymer through a GFLG linker sensitive to 
cathepsin B, a lysosomal cysteine protease overexpressed in several tumor types. ${ }^{23}$ Resulting NPs were non-fluorescent due to the self-quenching effect of Cy5. However, in the presence of cathepsin B, the cleavage of the linker led to Ptx and $\mathrm{Cy} 5$ release, concomitant with the emission of a fluorescence signal. After NP intravenous injection in 4T1 tumor-bearing mice, in vivo fluorescence imaging confirmed the linker cleavage in tumors.-A disulphide bond was used to design a NIR prodrug by linkage of dicyanomethylene-4H-pyrane (DCM), a NIR fluorophore, to Cpt (DCM-SS-CPT) and the conjugate was then encapsulated into PEG-PLA micelles. ${ }^{24}$ Drug release and simultaneous NIRF emission was observed in vitro after reductive cleavage of the disulfide bond (GSH $2.5 \mathrm{mM}$ ). Then, in vivo drug release in the reductive cytosolic conditions was monitored by NIRF and these redox responsive NPs displayed a higher antitumor activity compared to the free drug in human breast cancer BCap-37 tumor xenograph model.

Fluorescent ruthenium(II) polypyridyl complexes have been used as light-sensitive mesoporous silica nanoparticles (MPSN) capping system and fluorescent reporters of ruthenium interaction with its target. ${ }^{25}$ Upon visible light irradiation, the replacement of the monodentale ligand, which anchored the complex to the NP pores with a water molecule, led to the release of a nonfluorescent aqua ruthenium (II) complex. Fluorescence was then recovered after interaction with DNA due to the absence of water in such closely packed molecular superstructure. When Ptx was loaded in the pores, irradiation triggered the concomitant release of the two drugs.

\section{Multimodal imaging nanotheranostics}

Combination of more than one imaging modality in a single nanotheranostic has been investigated by several groups. For instance, Li et al. described liposomes made of DSPC/cholesterol/Gd-DOTA-DSPE/DOTA-DSPE, fluorescently labeled by insertion of the IRDye-DSPE in the bilayer and loaded with Dox. ${ }^{26}$ The Gd-DOTA-DSPE component endowed 
the liposome with magnetic resonance imaging (MRI) contrast enhancement properties, whereas the DOTA-DSPE enabled surface radiolabeling with ${ }^{64} \mathrm{Cu}$ for positron emission tomography (PET) imaging. Single-photon emission computed tomography (SPECT) imaging could also be realised by loading ${ }^{99} \mathrm{~m} \mathrm{Tc}$. Intratumoral injection of these multifunctional liposomes in a squamous cell carcinoma of head and neck xenograft model in rats was monitored by magnetic resonance (MR), NIRF, SPECT or PET imaging. MR images $\left(\mathrm{T}_{1}\right.$ or $\mathrm{T}_{2-}$ weighted) provided a 3D high-resolution microdistribution of the liposomes in the tumor, whereas optical imaging was used for detecting the locoregional retention and systemic distribution at superficial areas of the body, and nuclear images accurately measured the quantitative biodistribution of administrated radioactivity in vivo. Nevertheless, despite the Dox encapsulation, no proof of the therapeutic efficacy was provided and the use of this nanodevice as theranostic system still needs to be demonstrated.

MRI and optical imaging were also combined in peptide-functionalised Pluronic ${ }^{\circledR}$ L-121 polymersomes loaded with Cpt $(0.4 \mathrm{wt} \%)$ and superparamagnetic iron oxide NPs (SPIONs, 6 $\mathrm{nm}$ ) and surface decorated with a fluorescent dye. ${ }^{27}$ Compared to the free Cpt, drug-loaded polymersomes showed enhanced cytotoxic activity against prostate cancer cells (PC-3). Specific cell binding and internalization were confirmed by flow cytometry and confocal microscopy. In addition, their high transverse relaxivity $\left(r_{2}=394 \mathrm{~s}^{-1} \cdot \mathrm{mM}^{-1}\right)$ enabled to generate significant $\mathrm{T}_{2}$-weighted MRI contrast.

Association of optical and ${ }^{19} \mathrm{~F}$ MRI detection was also proposed with celecoxib-loaded perfluoro poly (ethylene glycol) ether nanoemulsion decorated with NIR fluorescent dyes (Cellvue ${ }^{\circledR}$ NIR815 or Cellvue ${ }^{\circledR}$ Burgundy). ${ }^{28}$ The nanoemulsion droplets suppressed the cyclooxygenase-2 (COX-2) activity in macrophages and could potentially be used in vivo to track macrophage tumor infiltration. 


\section{Mesoporous silica-based nanotheranostics}

Mesoporous silica nanoparticles (MSNPs) have robust and well-defined structures with excellent biocompatibility and biodegradability thanks to the absence of heavy elements and toxic degradation products. These particles can be easily modified by tuning their surface properties and their porosity thus enabling to achieve high host molecule payloads. ${ }^{29}$ MSNPs having intrinsic NIR photoluminescence, attributed to quantum confinement effect, were firstly applied by Sailor et al. for in vivo monitoring of both $(i)$ accumulation in tumor, liver and spleen and (ii) degradation to soluble silicic acid. ${ }^{30}$ Electrostatic interactions between Dox and the negatively charged porous $\mathrm{SiO}_{2}$ surface allowed preparing drug-loaded MSNPs which exhibited a similar or slightly greater cytotoxicity compared to the free drug in vitro on MDAMB-435 human breast carcinoma cells. The in vivo biodistribution study of MSNPs performed by whole-body fluorescence imaging showed that a dextran coating was efficient to slow down the degradation of the particles.

More recently, luminescent MSNPs were prepared by calcination which led to the generation of emitting carbon dots (CDs) in the particle matrix. ${ }^{31} \mathrm{c}(\mathrm{RGDyK})$ functionalised MPSNs were injected intravenously in U87MG tumor-bearing mice and distribution as well as tumor accumulation were monitored by in vivo whole body fluorescence imaging. Despite the fact that Dox was loaded in these NPs (70 wt $\%$ ), no proof of the antitumor activity of such NPs was provided.

Another kind of MSNPs with unique NIR-to-vis upconverting luminescence imaging properties (excitation at $980 \mathrm{~nm}$ ) and high drug loading of water-insoluble drugs was prepared by embedding Si nanocrystals in the mesoporous wall of the NPs and encapsulating graphite-like carbon in the mesopores. ${ }^{13}$ CTP was loaded into the pores of the same MSNPs, whose surface was then modified with PEG chains and hyaluronic acid (HA) for selective targeting of the CD44 receptors overexpressed on several cancer cells. Important cytotoxicity was observed in 
vitro on CD44 expressing MDA-MB-468 breast cancer cells and NIR-to-vis luminescence imaging enabled to simultaneously monitor the NP uptake.

MR, optical and drug delivery properties were combined in rodlike nanostructures (length 200 $\pm 23 \mathrm{~nm}$; diameter: $85 \pm 8 \mathrm{~nm}$ ) composed of a large hollow core and a double layered shell (inner magnetic shell and mesoporous outer shell) coated with rhodamine B (Rho B), and loaded with docetaxel or $\mathrm{Cpt}^{32}$ Drug loaded NPs exhibited greater cytotoxicity compared to the free drug in vitro on HeLa cancer cells and the fluorescent properties enabled to monitor and quantify uptake by flow cytometry and confocal microscopy. In addition, due to their high transverse relaxivity $\left(r_{2}=124.3 \mathrm{mM}^{-1} \cdot \mathrm{s}^{-1}, 3 \mathrm{~T}\right)$, it was possible to follow by MRI the NP biodistribution after intravenous injection in mice $(2.5 \mathrm{mg} \mathrm{Fe} / \mathrm{kg}$ body weight).

\section{Semiconductor quantum dots-based nanotheranostics}

Semiconductor quantum dots (QDs) exhibit unique optical and electronic properties due to quantum-confined structures. They have tunable fluorescence emission from visible to IR wavelengths controlled by their size (1-10 nm) and composition, broad absorption with narrow photoluminescence spectra, large absorption coefficients, high quantum yield and good photostability. ${ }^{33}$ Due to these properties they have been successfully used as traceable probes in several nanotheranostics. ${ }^{4}$ Although their intrinsic toxicity (especially when containing $\mathrm{Cd}$ and $\mathrm{Pb}$ ) has limited clinical translation, ${ }^{34}$ recent developments claimed the preparation of biocompatible QD formulations adapted for in vivo applications. ${ }^{4}$ But this would deserve a more convincing demonstration.

As example, CdSe/ZnS core-shell QD490 were conjugated with A10 RNA aptamers, recognizing the extracellular domain of the prostate specific membrane antigen (PSMA), and Dox was loaded in the double-stranded stem of the A10 aptamer (intercalation into the GC sequences).${ }^{35}$ This assembly resulted in a reversible self-quenching system based on a Bi-FRET 
mechanism (i.e., donor-acceptor Förster resonance energy transfer (FRET) model between QD and Dox and donor-quencher model FRET between Dox and aptamer) which led to a recovery of Dox fluorescence only when Dox was released. Cellular uptake of the QDs through PSMA binding and Dox release (either by physical dissociation or degradation of the aptamer) was observed after incubation within LNCaP prostate cancer cells. Cytotoxic activity of QDs was equivalent to the free drug.

Mucin1 (MUC1) aptamer specific for mutated MUC1 mucin, overexpressed in many cancer cells, was used by Minko et al. for functionalization of QDs to which Dox was conjugated via a pH-sensitive acyl hydrazone bond ${ }^{36}$ Fluorescence of conjugated Dox was quenched due to a FRET process between the QD and the drug and then recovered after its release $(35 \%$ in $5 \mathrm{~h}$ at pH 5.0). Functionalized Dox-loaded QD displayed higher cytotoxicity compared to the free drug and to their non-functionalized counterpart in multidrug resistant A2780-AD human ovarian cancer cells in vitro. Greater accumulation in the tumor was also observed after systemic administration in mice model of A2780-AD tumor xenograft (Figure 1).

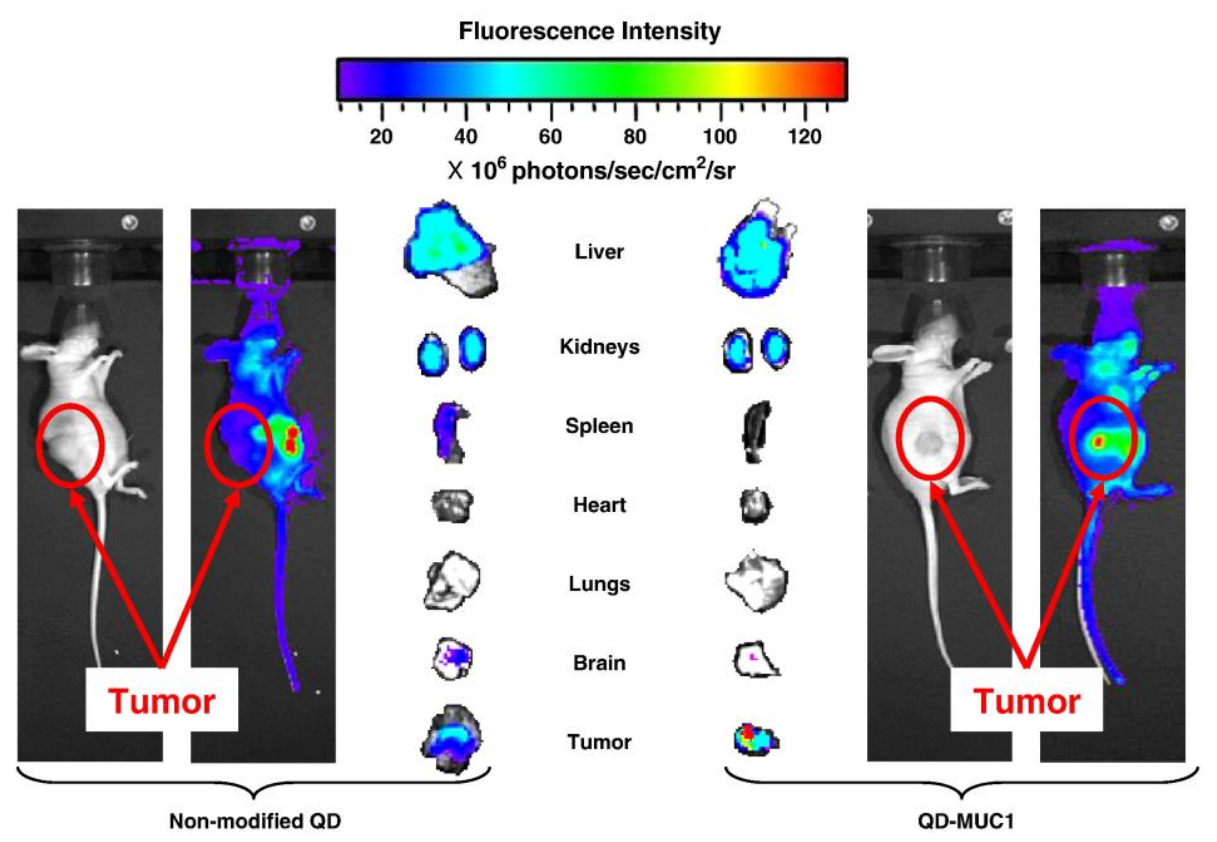

Fig. 1. Organ and tumor content of non-modified quantum dots (QD) and aptamer-targeted QD (QD-MUC1). Multidrug resistant A2780/AD cells were injected subcutaneously into the flanks 
of nude mice. The distribution of QD and QD-MUC1 in the whole animal was observed in realtime $24 \mathrm{~h}$ after intravenous injection using the IVIS imaging system. Immediately after mice were sacrificed and ex vivo imaging was the performed on excised organs. ${ }^{36}$ Reprinted with permission from ref 36. Copyright 2011 Elsevier B.V..

QD were also associated with lipid-based formulations, and encapsulated in the hydrophobic core of DSPE-PEG micelles ${ }^{37}$ or inserted in the bilayer of liposomes with different properties (i.e., cationic, PEGylated or deformable). ${ }^{38}$ When these formulations were loaded with Cpt or irinotecan, the highest cytotoxicity (in vitro on B16F10 cells) and greater in vivo tumor accumulation were observed with the cationic formulations (intratumoral injection). ${ }^{38}$ Interestingly, encapsulation in liposomes enabled to increase the tumor accumulation of $\mathrm{Cpt}$ but not that of irinotecan, as compared to free drugs.

To prepare theranostic thermosensitive nanogels, $\mathrm{Bi}_{2} \mathrm{O}_{3}$ QDs were immobilized inside PVA nanogels encapsulating temozolomide (TMZ) as therapeutic agent (DNA alkylating prodrug). ${ }^{39}$ Such nanogels undergo a reversible temperature-triggered volume phase transition which led to (i) a modification of the physicochemical microenvironment of the QDs and a variation of the fluorescence signal and (ii) a controlled release of TMZ $\left(7 \%\right.$ at $37{ }^{\circ} \mathrm{C} v s 19 \%$ at $40.0{ }^{\circ} \mathrm{C}$ for $24 \mathrm{~h})$. Such responsiveness was exhibited in response to variation of temperature over a physiological range $\left(37-40^{\circ} \mathrm{C}\right)$. Fluorescence and dark field imaging allowed to monitor the uptake of nanogels by mouse melanoma B16F10 cells. However, due to sustained drug release, these thermosensitive nanodevices displayed lower cytotoxicity compared to the free drug. Another strategy relied on the localization of QD at the surface of the drug carrier. For instance, layers of negatively charged QDs and positively charged poly(allylamine hydrochloride) (PAH) polyelectrolytes were used to coat Dox-loaded magnetic mesoporous silica-based ellipsoidal nanostructures $\mathrm{Fe}_{3} \mathrm{O}_{4} @ \mathrm{SiO}_{2} @ m \mathrm{SiO}_{2} .^{40}$ Thanks to the presence of the silica, no quenching of 
QD fluorescence occurred. It was possible to observe the intracellular localization of the nanostructures after incubation with MCF7 cells, when higher cytotoxicity was monitored comparatively to free Dox. Possibility of MRI detection of the nanostructures $\left(r_{2}=143 \mathrm{mM}^{-1} \cdot \mathrm{s}^{-}\right.$ ${ }^{1}$ at $3 \mathrm{~T}$ ) was also validated after subcutaneous injection into mouse.

\section{Lanthanide-doped upconversion nanotheranostics}

Lanthanide (Ln)-doped upconversion nanoparticles (UCNPs) have received considerable attention in bioimaging application due to their significant advantages over other optical imaging systems. UCNPs are constituted of a host material (or host lattice) (such as $\mathrm{NaYF}_{4}$, $\mathrm{Y}_{2} \mathrm{O}_{3}$ or $\mathrm{G}_{2} \mathrm{O}_{3}$ ) which is doped with one or more $\mathrm{Ln}$ ions (dopant or activator ions) (e.g., $\mathrm{Er}^{3+}$ (green light) or $\mathrm{Tm}^{3+}$ (blue light)). ${ }^{41} \mathrm{UCNPs}$ convert long wavelength radiation (NIR) to shorter wavelength light (visible/UV) through sequential NIR photon absorption or non-radiative energy transfer from a sensitizer (such as $\mathrm{Yb}^{3+}$ ) to the $\mathrm{Ln}$ ion. Emission profiles of UCNPs are tailored by energy level transitions of the Ln ions.

NIR light sensitivity allows fluorescence imaging in relatively deep tissues $(\sim 3 \mathrm{~cm})$ with a high signal-to-noise ratio, due to the low tissue scattering and absorption at these wavelengths. Additionally, UCNPs exhibit exceptional photostability, narrow emission peaks, long emission life time, tuneable emissions and large anti-stokes shifts compared to the conventional organic fluorescent dyes. Interestingly, UCNPs show lower cytotoxicity than $\mathrm{QD}^{42-44}$ and their luminescence signal is several orders of magnitude much stronger. PEGylation was proposed to increase the UCNP biocompatibility. ${ }^{45}$ Theranostic Ln-doped downconversion NPs (converting high energy photons - UV light - to low energy photons - visible or NIR light) were also reported but they display limited biological applications due to the low penetration of UV photons. ${ }^{46-49}$ 
For instance, intense green light was emitted under $980 \mathrm{~nm}$ NIR irradiation of $\mathrm{Yb}(\mathrm{OH}) \mathrm{CO}_{3} @ \mathrm{YbPO}_{4}: \mathrm{Er}^{3+}$ Dox-loaded UCNPs and the signal progressively increased with the drug release which reduced the quenching effect mediate by the encapsulated drug. Incubation of HeLa cells with these NPs was monitored by fluorescence imaging, revealing their uptake which resulted in greater cytotoxicity compared to the free drug. Anticancer molecules other than the Dox were also loaded in UCNPs. The anionic drug fluorouracil (5FU) was intercalated among layers of positively charged MgAl-layered double hydroxide nanosheets deposited on the surface of $\mathrm{Y}_{2} \mathrm{O}_{3}: \mathrm{Er}^{3+}, \mathrm{Yb}^{3+} @ \mathrm{SiO}_{2}$ nanospheres. ${ }^{50}$ Luminescent properties were validated and a higher cytotoxicity on MCF-7 cells was obtained compared to the free drug, possibly due to the positively charged surface of the UCNPs. In another study, CisPt(IV) and Rho B were conjugated to mPEG- $b$-PCL- $b$-PLL and then loaded in oleic acid-stabilized UCNPs. ${ }^{51}$ These UCNPs@Pt-RhoB were efficiently internalized in HeLa cells and the loaded platinium Pt(IV) prodrug was reduced to the active Pt(II), leading to higher apoptosis compared to both the free drug and the polymer prodrug. Antitumor activity was also confirmed also in vivo on SKOV-3 ovarian carcinoma bearing mice. The proof of concept of their optical detection was, however, only provided in C. elegans. Since trans-Pt(IV) prodrugs could be activated by UV, blue and green light, such an approach could represent an efficient strategy to decrease Pt(II) toxicity in healthy tissues. ${ }^{52,53}$

High stability, good dispersion in biological media and unique porous structure were achieved preparing UCNPs by one step synthesis using nucleotide-Ln ion supramolecular networks as precursors, rather than the conventional method (i.e., transfer from organic solvent) ${ }^{54}$ Different nucleotides (i.e., GMP, AMP, UMP, CMP) were used and the charged phosphate played an essential role in the preparation of porous UCNPs in which Dox was loaded. The theranostic features of such NPs were confirmed in vitro in A549 lung carcinoma cells. Other modifications 
of the preparation method included (i) the replacement of the source of fluoride host material $\left(\mathrm{NaBF}_{4} v s \mathrm{HF}\right)^{55}$ and (ii) electron beam irradiation of the solid particles, ${ }^{56}$ in order to achieve a more environmentally friendly or one-step synthesis, respectively. Dox was loaded in the resulting NPs which maintained their upconversion properties enabling to monitor the cell uptake.

In other systems, the UCNPs were used as a mediator to absorb the NIR photons and convert them into high energy photons (from the UV to the NIR region) used to trigger drug release ${ }^{57,58}$ or to promote cell targeting. ${ }^{59}$ For instance, Dox-loaded PEGylated $\mathrm{NaYF}_{4} \mathrm{Yb}, \mathrm{Tm} @ \mathrm{SiO}_{2}$ UCNPs were functionalized with caged FA using the $o$-nitrobenzyl photolabile group. ${ }^{59}$ CagedFA-PEGylated UCNPs@ $\mathrm{SiO}_{2}$-Dox were intravenously injected into mice bearing HeLa tumors and the tumor region was irradiated $\left(1 \mathrm{~h}, 980 \mathrm{~nm}, 1.8 \mathrm{~W} \cdot \mathrm{cm}^{-2}\right)$. Monitoring of the upconversion NIR emission confirmed that NIR irradiation was required for on-demand tumor targeting and UCNPs were found to accumulate at the tumor site during the first $5 \mathrm{~h}$ after injection. Moreover, with Caged-FA-PEGylated UCNPs@ $\mathrm{SiO}_{2}$-Dox NPs tumor growth was significantly inhibited as compared to the free drug, the irradiated non-targeted UCNP and the non-irradiated cagedFA UCNP.

\section{Combination of upconversion luminescence (UCL) and other imaging properties}

Alkali ion doped $\mathrm{CaF}_{2}: \mathrm{Yb}^{3+}, \mathrm{Er}^{3+} \mathrm{UCNPs}$ were reported for UCL/computed tomography (CT) imaging. ${ }^{60}$ Ln-doped $\mathrm{CaF}_{2} \mathrm{UCNPs}$ are more biocompatible than the $\mathrm{NaYF}_{4}$-based counterparts due to the high stability and non-hygroscopic behaviour of $\mathrm{CaF}_{2}$. These UCNPs display excellent NIR to visible upconversion ability and the presence of alkali ions in the fluoride crystal lattice influences the $\mathrm{Yb}^{3+}-\mathrm{Er}^{3+}$ distance, thus tuning the energy transfer and the $\mathrm{Ln}$ emission. For instance, a $\mathrm{CaF}_{2}$ shell was used to enhance the emission properties of $\mathrm{CaF}_{2}: \mathrm{Yb}^{3+}$, 
$\mathrm{Er}^{3+}, \mathrm{Cs}^{+} @ \mathrm{CaF}_{2} \mathrm{UCNPs}$ which were then coated with oleic acid and $\alpha-\mathrm{CD}$ to obtain a hydrophilic system. Uncharged Dox was loaded in the hydrophobic oleic acid layer and quickly released in acidic conditions as a result of Dox protonation and weakening of hydrophobic interactions. Dox-loaded UCNPs showed higher cytotoxicity compared to the free drug in vitro in A549 and HeLa cells. Interestingly, when these UCNPs were injected in the foot, backside and thin muscle of mouse (up to $5 \mathrm{~mm}$ depth), the green UCL emission under $980 \mathrm{~nm}$ NIR laser could be observed by naked eyes from the three regions. Due to the high degree of X-ray absorption of $\mathrm{Yb}$, these NPs displayed higher sensitivity as CT contrast agent properties than the commercial iopromide 300 .

MRI contrast properties were also combined to UCL by using the $\mathrm{NaGdF}_{4}$ as coating or core of different Dox loaded-UCNPs. ${ }^{61-64}$ As example, Dox loaded $\mathrm{NaYF}_{4}: \mathrm{Tm} / \mathrm{Yb} @ \mathrm{NaGdF}_{4}$ core/hollow mesoporous silica shell UCNPs were prepared and quenching of UCNPs emissions at $450 \mathrm{~nm}$ and $477 \mathrm{~nm}$ was observed due to luminescence resonance energy transfer (LRET) between the donor UCNPs and the acceptor Dox. ${ }^{62}$ In addition, the reduced probability of water molecules to bind to the $\mathrm{Gd}^{3+}$ ions led to a significant drop in the longitudinal relaxation $r_{1}$ value (from 12.29 to $2.96 \mathrm{mM}^{-1} \cdot \mathrm{s}^{-1}$ at $3 \mathrm{~T}$ ). Dox release upon $980 \mathrm{~nm}$ light excitation led to the recovery of both UCL and MR signals, which showed excellent linear relationship with the amount of released drug, allowing for quantitative measurements. Imaging features were validated in HeLa cells and in Zebrafish embryos after UCNPs injection in heart ventricles (51\% enhancement in the $\mathrm{T}_{1}$-MR signal intensity) (Figure 2). Analogue signal recovery was observed when Dox was replaced by CisPt, and monitored in vivo by MRI in walker-256 tumorbearing rats (Figure 2). Despite the theranostic potential of this system, its therapeutic ability is not yet demonstrated and still needs further investigation. 


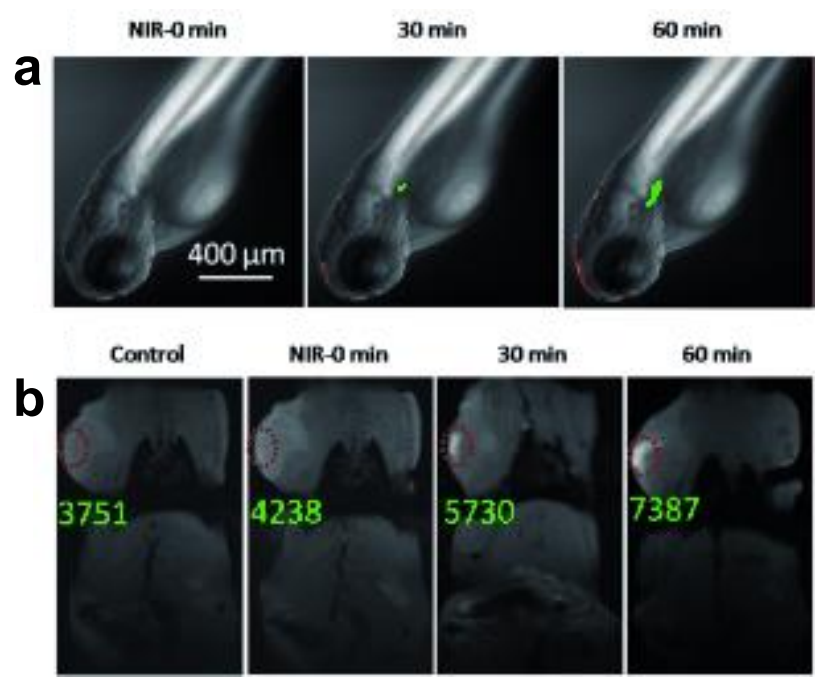

Fig. 2. a) CLSM images of zebrafish, (green and red correspond to UCNP and Dox signals respectively). b) $\mathrm{T}_{1}$-MR images of a walker-256 tumor-bearing Sprague Dawley rat injected with CisPt-UCNPs before and after NIR exposure for different time durations. Red circles indicate the tumor area where CisPt-UCNPs were injected. Green values indicate $\mathrm{T}_{1}$-MR signal intensities before and after NIR exposure. The power density of NIR light employed was 1.5 W.cm ${ }^{-2}{ }^{62}$ Reprinted with permission from ref 61. Copyright 2014 WILEY-VCH Verlag GmbH \& Co. KGaA, Weinheim.

Lin et al. used PEG coated $\mathrm{NaYF}_{4}: \mathrm{Yb}^{3+} / \mathrm{Tm}^{3+} @ \mathrm{NaGdF}_{4} / \mathrm{Yb}^{3+} \mathrm{UCNPs}(65 \mathrm{~nm})$ to convert the NIR light (980 nm) into UV (290-370 nm) and activate a dicarboxyl light-sensitive trans-Pt(IV) prodrug trans,trans,trans- $\left[\mathrm{Pt}\left(\mathrm{N}_{3}\right)_{2}\left(\mathrm{NH}_{3}\right)(\mathrm{py})\left(\mathrm{O}_{2} \mathrm{CCH}_{2} \mathrm{CH}_{2} \mathrm{COOH}\right)_{2}\right] \quad$ (DPP) covalently conjugated on the surface of the UCNP-PEG (26 mg Pt/UCNP-DPP-PEG g) ${ }^{65}$ Although very complex, this system combines drug delivery with UCL, MR $\left(\mathrm{Gd}^{3+}\right.$ ions) and CT $\left(\mathrm{Gd}^{3+}\right.$ and $\mathrm{Yb}^{3+}$ ) imaging properties, which allows precise monitoring of NPs after intratumoral and intravenous injection. (Figure 3). UCNP-DPP-PEG (0.65 mgPt/kg body weight) intratumorally injected displayed a significant inhibition of tumor growth in $\mathrm{H} 22$ hepatic tumor-bearing mice after irradiation at $980 \mathrm{~nm}$, while only reduced anticancer activity was observed when irradiated at $365 \mathrm{~nm}$, due to the lower tissue penetration of UV light. Other trimodal imaging particles loaded with Dox via a pH sensitive hydrazone bond were also reported. ${ }^{66}$ 


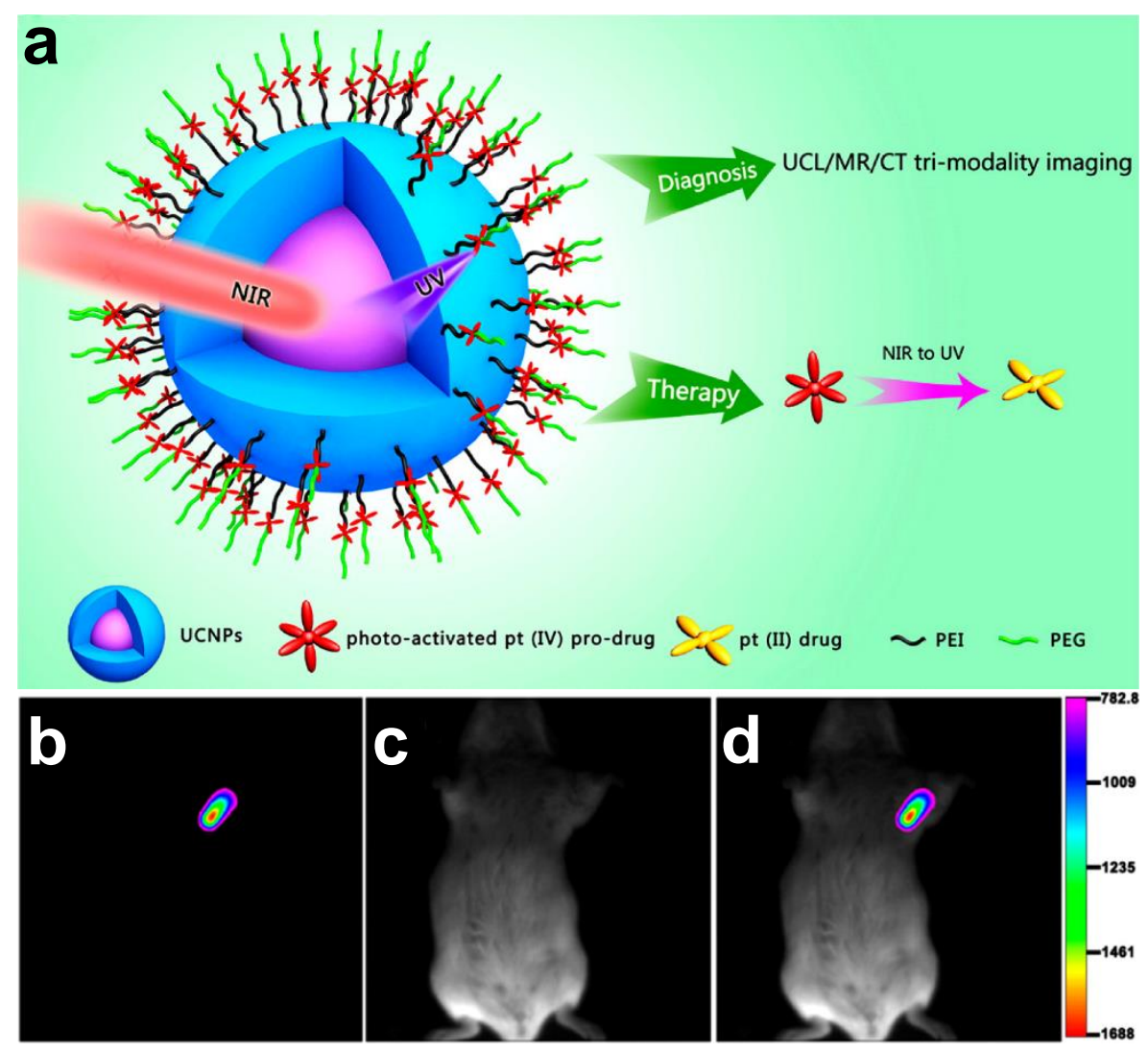

Fig. 3. (a). Schematic illustration of UCNP-DPP-PEG NPs (b-d). In vivo UCL imaging of a tumor-bearing Balb/c mouse after injection of UCNPs at the tumor site: UCL (b), bright field (c), and overlay images (d). ${ }^{65}$ Adapted with permission from ref 64. Copyright 2013 American Chemical Society.

UCL and magnetic properties (allowing magnetic guidance by an external magnetic field) were exhibited by $\mathrm{Fe}_{3} \mathrm{O}_{4} @ \mathrm{mSiO}_{2} @ \alpha-\mathrm{NaYF}_{4}: \mathrm{Yb}^{3+}, \mathrm{Er}^{3+}$ nanorattles, consisting of a silica-coated magnetite inner core coated with hollow mesoporous silica and layers of $\mathrm{Yb}^{3+} / \mathrm{Er}^{3+}$-doped cubic $\alpha-\mathrm{NaYF}_{4}{ }^{67}$ Application of the magnetic field increased cell accumulation and cytotoxicity of Dox-loaded nanorattles in vitro in human hepatoma cells (QGY-7703), as compared to without magnetic field; enhanced tumor accumulation in vivo in $\mathrm{H} 22$ tumor-bearing mice was also observed after monitoring by UCL whole-body imaging. Such increased tumor accumulation resulted in higher tumor suppression (96\%) compared to nanorattles without magnetic guidance $(53 \%)$ and to the free drug $(35 \%)$. 


\section{Gold containing nanotheranostics}

Gold NPs exhibit tunable localized surface plasmon resonance (SPR), which allows to achieve deep-tissue imaging in the light-transparent NIR biological window $(700-950 \mathrm{~nm})$ and/or localised temperature increase. This photothermal effect can be exploited for hyperthermal therapy and/or to trigger the release of drugs from gold-containing drug delivery systems. In addition, gold-based systems can act as imaging agents in combination with various fluorophores. Such approach could be used to detect variation of the fluorophore/gold distance, on the basis of the fluorescence quenching effect of gold, and therefore provide useful information on nanocarrier biodistribution and related drug delivery efficiency. ${ }^{68}$

Various approaches have been adopted to prepare gold containing nanotheranostics. For instance, PEG-PLGA-Au half shell NPs were developed to simultaneously actuate chemotherapy (Dox loading) and hyperthermia upon NIR irradiation. ${ }^{69}$ Synergistic effect was observed in A431 human epidermoid carcinoma tumor-bearing mice after NIR irradiation (2.56 W. $\mathrm{cm}^{-2}$ for $10 \mathrm{~min}$, generating an intratumoral temperature increase to $50-70{ }^{\circ} \mathrm{C}$ ). This protocol led to a complete destruction of the tumor 7 days after intravenous injection of the NPs whose tumor accumulation was monitored by in vivo whole body NIR imaging. Complete gold coating of Dox-loaded PLGA NPs and liposomes was also proposed..$^{70,71}$

As an alternative strategy, bovine serum albumin (BSA) stabilised gold clusters were encapsulated into polymer NPs composed of mPEG-PLGA and $\varepsilon$-polylysine. ${ }^{72}$ Further NPs labelling with FITC enabled to monitor their tumor accumulation in B16-F10 melanoma bearing mice by in vivo two-color imaging. Co-loading of Dox (hydrophilic core), Ptx (hydrophobic layer) and surviving siRNA (external surface), targeting different essential metabolic pathways of tumor cells, resulted in a significant therapeutic efficacy. Interestingly, 
the surviving siRNA being released before the other two drugs, induced a sensitization effect playing a crucial role to obtain the strong antitumor activity.

Another original system consisted in amphiphilic gold NPs coated with BGLA Raman reporters and mixed polymer brushes of hydrophilic PEG and $\mathrm{pH}$ sensitive hydrophobic chains (Figure 4a). ${ }^{73}$ These amphiphilic gold NPs were self-assembled into plasmonic vesicles adapted for tumor-targeted drug delivery and monitoring of cargo release by plasmonic imaging and Raman spectroscopy. The vesicles were functionalised with the antiHER 2 antibody and loaded with Dox which was released at $\mathrm{pH} 5$ in late endosomes/lysosomes, due to the transition of hydrophobic brushes to hydrophilic ones, further triggering vesicles disassembly. Raman spectroscopy, dark field and fluorescence imaging allowed to independently track assembled/disassembled vesicles and Dox release, thus permitting to precisely monitor the fate of the plasmonic vesicles when incubated with HER2-positive SKBR-3 breast cancer cells. Vesicle cellular binding, vesicle disassembly, drug release in the cytosol and penetration in the nucleus were clearly visualized. (Figure $4 \mathrm{~b}$ ). To be noted that Dox-loaded targeted vesicles exhibited higher cytotoxicity compared to the non-targeted ones, with a 5-fold reduction of the $\operatorname{IC}_{50}(0.31 \mu \mathrm{g} / \mathrm{mL})$.

a

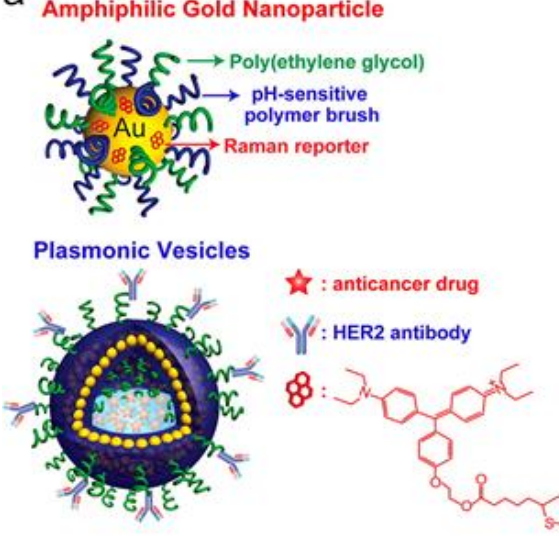

b

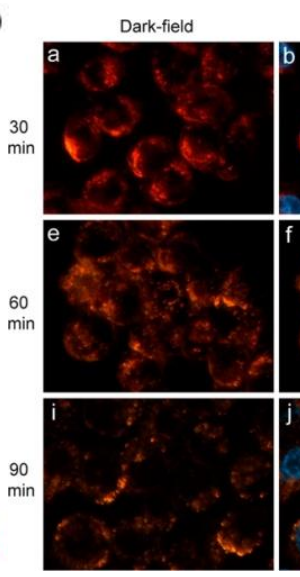

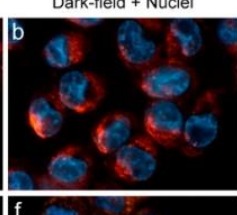

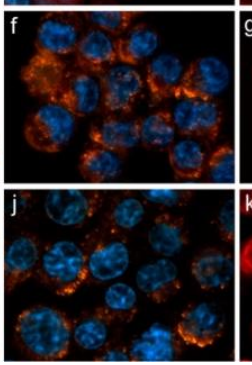

Fluorescence (DOX)

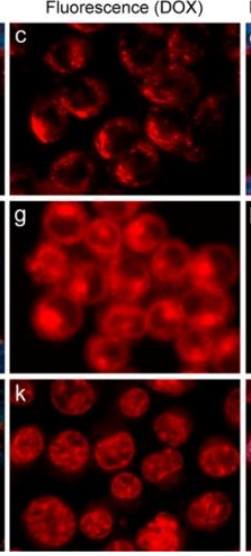

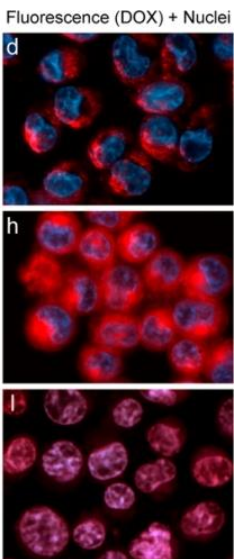

Fig. 4. (a) Schematic illustration of the amphiphilic gold NP and assembled plasmonic vesicle. (b) Dark-field, fluorescence and the overlaid images of SKBR-3 cells labeled with Dox-loaded $\mathrm{pH}$-sensitive plasmonic vesicles after 30,60 and $90 \mathrm{~min}$ incubation; Dox has a red fluorescence, and cell nuclei were counterstained with Hoechst 3342 exhibiting blue fluorescence. ${ }^{73}$ Adapted with permission from ref 72. Copyright 2012 American Chemical Society. 
In another study, hybrid nanogels composed of a silver-gold NP core, a shell made of thermoresponsive nonlinear PEG-based hydrogel and HA chains as targeting ligands, were loaded with TMZ. ${ }^{74}$ Temperature variations induced reversible swelling and shrinking of the gel providing modification of the physicochemical environment and of the mesh size in the gel network which affected the fluorescence of the silver-gold core and regulated the drug release. In addition, a synergistic effect of chemo and photothermal therapy was observed in vitro on B16F10 mouse melanoma cells upon NIR irradiation.

As an alternative to the loading of gold NPs into a supramolecular nanodevice, direct modification of the gold surface was proposed. For instance, spherical gold NPs were functionalised with PEG chains, biotin molecules and Rho B-linked $\beta$-cyclodextrins $(\beta-\mathrm{CD})$ used as Ptx pocket. ${ }^{75}$ Reductive condition (10 mM GSH) promoted the release of Ptx (via ligand exchange) and Rho B, which was associated with the emission of a strong fluorescence signal previously quenched by the proximity to the gold surface. Ligand-mediated uptake and cytotoxicity of these NPs were monitored by flow cytometry and confocal microscopy in Hela cancer cells. Electrostatic interactions were also used to load Dox at the surface of amine and carboxylic acid-terminated GNRs. ${ }^{76}$ Taking advantage of both GNR luminescence and Dox fluorescence, it was possible to monitor by multi-fluorescence lifetime imaging, GNR uptake (KB cancer cells), as well as drug release thanks to the reduced quenching effect of GNRs over Dox fluorescence. Surface of gold nanorods was also modified with a mesoporous silica layer ${ }^{77}$ or with bio-friendly C-dots. ${ }^{78}$ In both cases, the porous nature of the coating allowed Dox loading and a combination of chemo and photothermal therapy was observed upon NIR irradiation. Imaging properties of these systems enabled their tracking in vitro, in different cell lines. 


\section{Iron oxide-based theranostic nanoprobes}

Iron oxide nanoparticles (IONPs) have found wide clinical application as MRI contrast agents, thanks to their high transverse relaxivity $r_{2}$, which leads to a pronounced negative contrast on $\mathrm{T}_{2}$-weighted MR images (hypointensity). In addition, when exposed to an alternative magnetic field, they generate heat in the surrounding medium due to hysteresis loss and/or Néel relaxation. Therefore, they have been extensively used for selective increase of temperature in tumors, (i.e., hyperthermia). ${ }^{79}$ Opportune modifications of IONPs also enable to combine imaging features (fluorescence and MR) and magnetic guidance.

For instance, ultrasmall superparamagnetic iron oxide (USPIO) NPs were coated with the hydrophilic biocompatible $N$-phosphonomethyl iminodiacetic acid (PMIDA) and then aminederivatized in order to introduce Rho B, FA (targeting ligand) and methotrexate (therapeutic agent) via amide, thiourea and $\mathrm{pH}$-labile ester linkage, respectively.$^{80}$ In vitro experiments were performed on FA receptor expressing HeLa cells. NPs uptake as well as cytotoxicity, mediated by a faster intracellular drug release in acidic conditions, were assessed. In addition, NPs showed high transverse relaxivity $\left(r_{2}=295 \pm 7 \mathrm{~s}^{-1} \cdot \mathrm{mM}^{-1}, 0.3 \mathrm{~T}\right)$, allowing their use as imaging contrast agents.

In another approach, lipophilic NIR dyes (dialkylcarbocyanine fluorophore, DiI or DiR) and drugs (Ptx) were loaded together within the hydrophobic pockets of poly(acylic acid) (PAA) at the surface of IONPs ${ }^{81}$ NPs surface was further functionalized with FA via copper-catalyzed azide-alkyne cycloaddition (click chemistry) for cancer cell targeting. NPs were stable in buffer at $\mathrm{pH} 7.4$, while a fast drug release was observed in the presence of esterase or in acidic conditions ( $\mathrm{pH} 4.0$ ). Cytotoxicity of the NPs was demonstrated on the lung carcinoma A549 
cell line. Simultaneous MR $\left(r_{2}=202 \mathrm{~s}^{-1} \cdot \mathrm{mM}^{-1}, 0.47 \mathrm{~T}\right)$ and fluorescence imaging of NP uptake in folate-receptor expressing A549 cells was performed.

IONPs coating with phosphonic acid terminated poly(4- $O$-acryloyl benzaldehyde- $b$ oligoethylene glycol-acrylate) block copolymers provided both a hydrophilic, biocompatible layer at the IONP surface and functional groups for conjugation of Dox via $\mathrm{pH}$ sensitive Schiff base/imine bonds (IONP@P(HBA)- $b$-P(OEGA $)+$ Dox $).{ }^{82}$ Dox intracellular release and localization were precisely monitored in H1299 lung and MCF-7 breast cancer cells using fluorescence lifetime imaging microscopy (FLIM) coupled with Phasor plot analysis (Figure 5). Such technique detects changes in Dox fluorescence lifetime on the basis of its microenvironment, thus allowing to follow accumulation in the different intracellular compartments. Cell uptake of Dox-loaded IONPs in multicellular 3D tumor spheroids was also investigated by multiphoton excitation microscopy and increase of cytotoxicity comparatively to the free drug was observed. In addition, transverse relaxation of the IONPs was enhanced by $70 \%$ after Dox release $\left(r_{2}=223 \mathrm{~s}^{-1} \cdot \mathrm{mM}^{-1}\right.$ and $386 \mathrm{~s}^{-1} \cdot \mathrm{mM}^{-1}$ at $9.4 \mathrm{~T}$ before and after Dox release, respectively), which suggests their potential application for monitoring drug release by MRI.

a

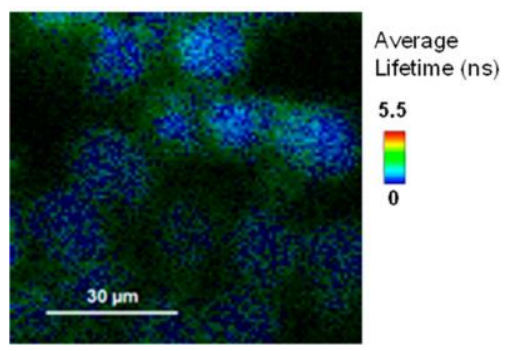

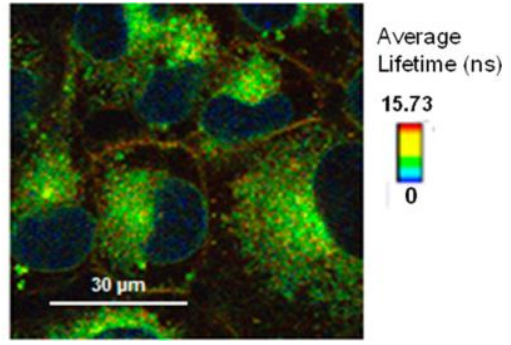

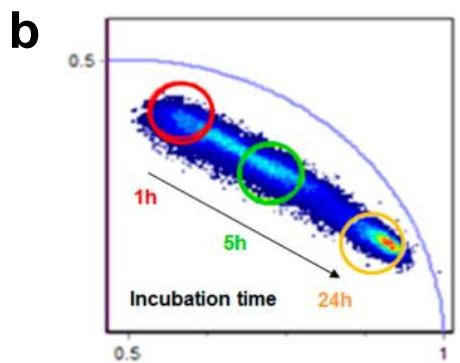

Fig. 5. (a) FLIM images showing cell uptake of native Dox (left) and IONP@P(HBA)-b$\mathrm{P}($ OEGA) + Dox (right) using H1299 cells after $5 \mathrm{~h}$ of incubation. (b) Phasor plot derived from the FLIM images at different incubation times with IONP@P(HBA)- $b$-P(OEGA) + Dox. Red region of interest (ROI) corresponds to the signal after $1 \mathrm{~h}$ of incubation; green ROI and orange ROI correspond to the signals after 5 and $24 \mathrm{~h}$, respectively. Each ROI corresponds to different lifetimes of Dox in the cells corresponding to different amounts of Dox released from IONP@P(HBA)- $b$-P(OEGA) + Dox. ${ }^{82}$ Adapted with permission from ref 81. Copyright 2013 American Chemical Society. 
Surface modification of iron oxide NPs was also realized using $(i)$ the Rotavirus capsid surface protein $\mathrm{VP} 4,{ }^{83}$ (ii) self-assembled DNA biopolymer, ${ }^{84}$ or $(\mathrm{iii})$ gold. ${ }^{85}$ The latter where further functionalized and combined in a system with five different functions: cancer cell targeting, MR and optical imaging, photothermal and chemotherapy. Derivatization of gold surface with carboxylic terminated PEG chains and a capture probe (5'-(GCT) 7 -T 10 -SH-3') allowed both the hybridization with the dye-modified sgc8 aptamer and the Dox loading via intercalation into the GC base pairs and electrostatic interaction with the carboxyl groups (Figure 6). ${ }^{85}$ Fluorescence imaging was used to monitor NPs cell uptake and Dox release. Drug release was promoted by NIR irradiation, leading to degradation of nucleotides and dehybridization of aptamer mediated by the localised increase of temperature, which also enabled to combine chemotherapy and photothermal therapy. Cytotoxicity on CCRF-CEM cells indeed increased upon laser irradiation. The magnetic core allowed to monitor cell uptake also by MRI $\mathrm{T}_{2}$ measurement.

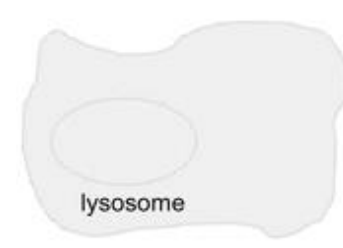

Cancer cells

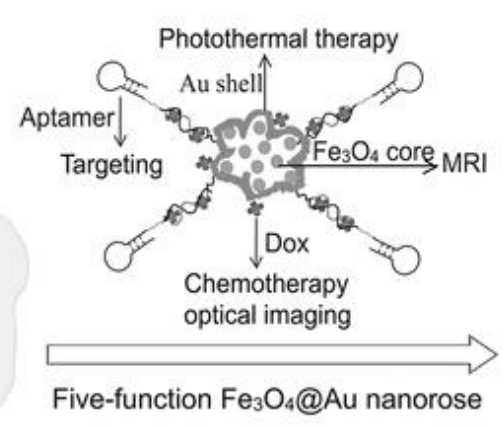

Five-function $\mathrm{Fe}_{3} \mathrm{O}_{4} @ \mathrm{Au}$ nanorose

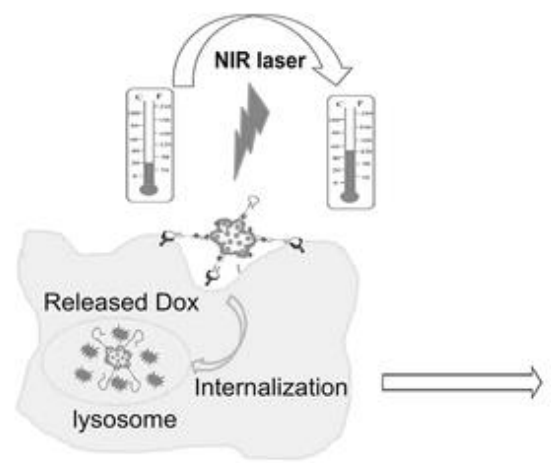

Imaging and therapy
Cell death

Fig. 6. Schematic representation of five-function $\mathrm{Fe}_{3} \mathrm{O}_{4} @ \mathrm{Au}$ nanorose for cancer cell targeting, MRI, optical imaging, photothermal and chemotherapy. ${ }^{85}$ Reprinted with permission from ref 81. Copyright 2013 WILEY-VCH Verlag GmbH \& Co. KGaA, Weinheim.

\section{Radioluminescent theranostic nanoprobes}

As previously discussed, the in vivo application of optical imaging is hindered by the tissue autofluorescence, light adsorption and scattering. Although better tissue penetration is obtained by NIR light irradiation, the detection of fluorescent probes is still limited to few centimetres 
and the resolution is very low. In this context, X-ray excited optical luminescence (XEOL) approaches were introduced recently to address these issues. These imaging modalities are based on the selective excitation and optical detection of X-ray excitable phosphor NPs, which emit NIR light when excited by X-ray photons. Thus, unprecedented high imaging resolution could be successfully achieved in deep tissues due to the very low background provided by this new technique. ${ }^{86-88}$

For instance, Anker et al. used XEOL to monitor drug delivery and biodistribution of a drug nanocarrier in vivo. ${ }^{89}$ Practically, spindle-shaped monodispersed silica nanocapsules (length of $420 \pm 20 \mathrm{~nm}$ and width of $130 \pm 15 \mathrm{~nm}$,) were loaded with Dox (5 wt\%) and coated with X-ray scintillator $\mathrm{Gd}_{2} \mathrm{O}_{2} \mathrm{~S}$ :Tb or $\mathrm{Gd}_{2} \mathrm{O}_{2} \mathrm{~S}$ :Eu and $\mathrm{pH}$ sensitive PSS/PAH multilayers. The X-ray absorption by the $\mathrm{Gd}_{2} \mathrm{O}_{2} \mathrm{~S}$ host lattice produced electron-hole pairs which excited the $\mathrm{Tb}^{3+}$ or $\mathrm{Eu}^{3+}$ centres, leading to the emission of visible or NIR light. The quenching effect of loaded Dox on specific emission wavelengths enabled to detect Dox release in vitro by monitoring the increase of X-ray luminescence intensity; faster release was observed at $\mathrm{pH} 5.0$ compared to pH 7.4. In vivo tracking of $\mathrm{Gd}_{2} \mathrm{O}_{2} \mathrm{~S}$ :Eu-coated nanocapsules was performed by monitoring luminescence after irradiation with a miniature X-ray source. The presence of the PSS/PAH layers led to a slower liver and spleen accumulation compared to the same but non-coated NPs. Interestingly, the presence of $\mathrm{Gd}^{3+}$ ions made these nanocapsules adapted for MRI $\left(r_{2} \sim 50 \mathrm{mM}^{-}\right.$ $\left.{ }^{1} \cdot \mathrm{s}^{-1}\right)$. Similarly, metroxantrone-loaded core shell NPs composed of $\mathrm{Gd}_{2} \mathrm{O}_{2} \mathrm{~S}: \mathrm{Eu}$ and $\mathrm{Yt}^{3+}, \mathrm{Tm}^{3+}$ were designed to combine the advantages of UCL, MR and radioluminescence imaging, which allowed submillimeter resolution through thick deep tissues $(>1 \mathrm{~cm}) .{ }^{90}$

Despite the great interest of these systems as imaging and drug delivery tools, their real theranostic potential still remains to be demonstrated, as no proof of their therapeutic activity has been described yet. 


\section{Miscellaneous}

PEI-PLA copolymers were used to prepare Ptx-loaded (5.11 wt \%) luminescent polymer dots (PDs) which possess ultrabright and multicolor fluorescent properties. ${ }^{91} \mathrm{pH}$-dependent variation of the PD structure rigidity was found to influence the fluorescence signal. Antitumor efficacy was assessed after intravenous injection of the PDs in MCF-7 tumor-bearing mice (compared to free Ptx) and ex vivo fluorescence confirmed the tumor accumulation.

High water solubility, easy surface modification, biocompatibility, good cell permeability, high photostability and excitation-dependent multicolor emission make carbon dots (CDs) particularly attractive for the design of nanotheranostics. ${ }^{92} \mathrm{CD}$ surface-modified with a Pt(IV) complex were intratumorally injected (2 injections spaced by $24 \mathrm{~h}$ ) in $\mathrm{H} 22$ hepatocarcinomabearing mice and tracked by in vivo fluorescence. Significant antitumor activity was observed, with a $82.4 \%$ reduction of tumor volume in $72 \mathrm{~h} .{ }^{93}$

\section{Conclusion}

Optically detectable nanotheranostics enabling sustained drug release were extensively studied in the last decades. A large variety of sophisticated nanoplatforms were proposed for providing and monitoring a therapeutic effect in real-time in order to adjust the treatment on-demand. However, in spite of the large variety of approaches proposed until now, challenges still remain to be overcome such as the improvement of detection in deep tissues, the direct monitoring of the therapeutic effect or the on-demand control of therapeutic action. To be noted that most of the studies were performed in vitro cell culture experiments. Even if the proof of concept of few of the described systems has been already done in preclinical animal models, administration was generally performed intratumorally, instead of intravenously which is the route of administration used in the clinical routine. Finally, the toxicological issues are generally not 
investigated following the usual guidelines for drug development. There is, therefore, plenty of room for further research on nanodevices for optical imaging and therapy.

\section{Lists of abbreviations}

2-(4-(bis(4-(diethylamino)phenyl)(hydroxy)methyl)phenoxy)ethyl

5-(1,2-dithiolan-3-

yl)pentanoate (BGLA), 6-(((4,4-difluoro-5-(2-thienyl)-4-bora-3a,4a-diaza-s-indacene-3yl)styryloxy)acetyl) aminohexanoic acid (BODIPY), bovine serum albumin (BSA), carbon dot (CD), $\beta$-cyclodextrins ( $\beta$-CD), confocal laser scanning microscopy (CLSM), cyclooxygenase2 (COX-2), camptothecin (Cpt), computed tomography (CT), Cyanine5.5 (Cy5.5), dicyanomethylene-4H-pyrane (DCM), 3,3'-dioctadecyloxacarbocyanine perchlorate (DiO), dioleoylphosphatidylethanolamine $\quad$ (DOPE), 1,4,7,10-tetraazacyclododecane-1,4,7,10tetraacetic acid (DOTA), Doxorubicin (Dox), trans,trans,trans$\left[\mathrm{Pt}\left(\mathrm{N}_{3}\right)_{2}\left(\mathrm{NH}_{3}\right)(\mathrm{py})\left(\mathrm{O}_{2} \mathrm{CCH}_{2} \mathrm{CH}_{2} \mathrm{COOH}\right)_{2}\right]$ (DPP), distearoylphosphatidylcholine (DSPC), distearoylphosphatidylethanolamine (DSPE), folic acid (FA), fluorescein isothiocyanate (FITC), fluorescence lifetime imaging microscopy (FLIM), Förster resonance energy transfer (FRET), glutathione (GSH), hyaluronic acid (HA), $N$-(2-hydroxypropyl) methacrylamide (HMPA), indocyanine green (ICG), iron oxide nanoparticle (IONP), lanthanides (Ln), luminescence resonance energy transfer (LRET), methoxy poly(ethylene glycol) (mPEG), mesoporous silica nanoparticles (MPSN), magnetic resonance (MR), magnetic resonance imaging (MRI), Mucin1 (MUC1), near-infrared (NIR), NIR fluorescence (NIRF), nanoparticle (NP), poly(acylic acid) (PAA), poly(allylamine hydrochloride) (PAH), poly( $\gamma$-benzyl Lglutamate) (PBLG), L- $\alpha$-phosphatidylcholine (PC), polymer dot (PD), poly(ethylene glycol) (PEG), poly(ethylene imine) (PEI), positron emission tomography (PET), egg L- $\alpha-$ phosphatidyl-DL-glycerol (PG), poly(D,L-lactide) (PLA), $N$-phosphonomethyl iminodiacetic acid (PMIDA), prostate specific membrane antigen (PSMA), poly(sodium 4-styrenesulfonate) 
(PSS), paclitaxel (Ptx), semiconductor quantum dots (QD), rhodamine B (Rho B), region of interest (ROI), single-photon emission computed tomography (SPECT), superparamagnetic iron oxide nanoparticle (SPION), surface plasmon resonance (SPR), temozolomide (TMZ), upconversion luminescence (UCL), Lanthanide-doped upconversion nanoparticle (UCNP), ultrasmall superparamagnetic iron oxide (USPIO), ultraviolet (UV), X-ray excited optical luminescence (XEOL).

\section{References}

1. M. S. Muthu, D. T. Leong, L. Mei and S.-S. Feng, Theranostics, 660 (2014).

2. G. Chen, J. Shen, T. Y. Ohulchanskyy, N. J. Patel, A. Kutikov, Z. Li, J. Song, R. K. Pandey, H. Agren, P. N. Prasad and G. Han, ACS Nano, 8280 (2012).

3. S. Srinivasan, B. W. Pogue, S. Jiang, H. Dehghani, C. Kogel, S. Soho, J. J. Gibson, T. D. Tosteson, S. P. Poplack and K. D. Paulsen, Proc. Natl. Acad. Sci. U. S. A., 12349 (2003).

4. K.-T. Yong, Y. Wang, I. Roy, H. Rui, M. T. Swihart, W.-C. Law, S. K. Kwak, L. Ye, J. Liu, S. D. Mahajan and J. L. Reynolds, Theranostics, 681 (2012).

5. J. Shen, L. Zhao and G. Han, Adv. Drug Delivery Rev., 744 (2013).

6. Y. Jin, Acc. Chem. Res., 138 (2014).

7. S. R. Rajski and R. M. Williams, Chem. Rev., 2723 (1998).

8. T. Thambi, H.-Y. Yoon, K.-M. Kim, I.-C. Kwon, C.-K. Yoo and J.-H. Park, Bioconj. Chem., 1924 (2011).

9. P. Mohan and N. Rapoport, Mol. Pharmaceutics, 1959 (2010).

10. J. R. Mourant, T. M. Johnson, G. Los and I. J. Bigio, Phys. Med. Biol., 1397 (1999).

11. G. M. Palmer, R. J. Boruta, B. L. Viglianti, L. Lan, I. Spasojevic and M. W. Dewhirst, J. Controlled Release, 457 (2010).

12. T. M. Baran and T. H. Foster, Lasers Surg. Med., 542 (2013).

13. Q. He, M. Ma, C. Wei and J. Shi, Biomaterials, 4392 (2012).

14. T. P. Gustafson, Y. H. Lim, J. A. Flores, G. S. Heo, F. Zhang, S. Zhang, S. Samarajeewa, J. E. Raymond and K. L. Wooley, Langmuir, 631 (2014).

15. S. Santra, C. Kaittanis and J. M. Perez, Mol. Pharmaceutics, 1209 (2010).

16. K. Kim, J. H. Kim, H. Park, Y.-S. Kim, K. Park, H. Nam, S. Lee, J. H. Park, R.-W. Park, I.-S. Kim, K. Choi, S. Y. Kim, K. Park and I. C. Kwon, J. Controlled Release, 219 (2010).

17. S. Santra and J. M. Perez, Biomacromolecules, 3917 (2011).

18. J. Y. Lee, D.-Y. Choi, M. Y. Cho, K. E. Park, S.-H. Lee, S. Hun Cho, K. S. Hong and Y. T. Lim, Small, 901 (2014).

19. E. A. Murphy, B. K. Majeti, L. A. Barnes, M. Makale, S. M. Weis, K. Lutu-Fuga, W. Wrasidlo and D. A. Cheresh, Proc. Natl. Acad. Sci. U. S. A., 9343 (2008).

20. X. He, M.-H. Na, J.-S. Kim, G.-Y. Lee, J. Y. Park, A. S. Hoffman, J.-O. Nam, S.-E. Han, G. Y. Sim, Y.K. Oh, I.-S. Kim and B.-H. Lee, Mol. Pharmaceutics, 430 (2011).

21. C.-K. Huang, C.-L. Lo, H.-H. Chen and G.-H. Hsiue, Adv. Funct. Mater., 2291 (2007).

22. B. S. Lee, K. Park, S. Park, G. C. Kim, H. J. Kim, S. Lee, H. Kil, S. J. Oh, D. Chi, K. Kim, K. Choi, I. C. Kwon and S. Y. Kim, J. Controlled Release, 253 (2010).

23. S. Ferber, H. Baabur-Cohen, R. Blau, Y. Epshtein, E. Kisin-Finfer, O. Redy, D. Shabat and R. Satchi-Fainaro, Cancer Lett., 81 (2014). 
24. X. Wu, X. Sun, Z. Guo, J. Tang, Y. Shen, T. D. James, H. Tian and W. Zhu, J. Am. Chem. Soc., 3579 (2014).

25. M. Frasconi, Z. Liu, J. Lei, Y. Wu, E. Strekalova, D. Malin, M. W. Ambrogio, X. Chen, Y. Y. Botros, V. L. Cryns, J.-P. Sauvage and J. F. Stoddart, J. Am. Chem. Soc., 11603 (2013).

26. S. Li, B. Goins, L. Zhang and A. Bao, Bioconj. Chem., 1322 (2012).

27. R. Bleul, R. Thiermann, G. U. Marten, M. J. House, T. G. S. Pierre, U. O. Haefeli and M. Maskos, Nanoscale, 11385 (2013).

28. S. K. Patel, Y. Zhang, J. A. Pollock and J. M. Janjic, PLoS One, e55802 (2013).

29. M. W. Ambrogio, C. R. Thomas, Y.-L. Zhao, J. I. Zink and J. F. Stoddart, Acc. Chem. Res., 903 (2011).

30. J.-H. Park, L. Gu, G. von Maltzahn, E. Ruoslahti, S. N. Bhatia and M. J. Sailor, Nat. Mater., 331 (2009).

31. H. Chen, Z. Zhen, W. Tang, T. Todd, Y.-J. Chuang, L. Wang, Z. Pan and J. Xie, Theranostics, 650 (2013).

32. H. Wu, S. Zhang, J. Zhang, G. Liu, J. Shi, L. Zhang, X. Cui, M. Ruan, Q. He and W. Bu, Adv. Funct. Mater., 1850 (2011).

33. A. P. Alivisatos, Science, 933 (1996).

34. A. M. Derfus, W. C. W. Chan and S. N. Bhatia, Nano Lett., 11 (2004).

35. V. Bagalkot, L. Zhang, E. Levy-Nissenbaum, S. Jon, P. W. Kantoff, R. Langer and O. C. Farokhzad, Nano Lett., 3065 (2007).

36. R. Savla, O. Taratula, O. Garbuzenko and T. Minko, J. Controlled Release, 16 (2011).

37. R. Kumar, A. Kulkarni, D. K. Nagesha and S. Sridhar, Theranostics, 714 (2012).

38. C.-J. Wen, C. T. Sung, I. A. Aljuffali, Y.-J. Huang and J.-Y. Fang, Nanotechnology, 325101 (2013).

39. H. Zhu, Y. Li, R. Qiu, L. Shi, W. Wu and S. Zhou, Biomaterials, 3058 (2012).

40. Y. Chen, H.-R. Chen, S.-J. Zhang, F. Chen, L.-X. Zhang, J.-M. Zhang, M. Zhu, H.-X. Wu, L.-M. Guo, J.-W. Feng and J.-L. Shi, Adv. Funct. Mater., 270 (2011).

41. D. J. Naczynski, M. C. Tan, R. E. Riman and P. V. Moghe, J. Mater. Chem. B, 2958 (2014).

42. R. J. Palmer, J. L. Butenhoff and J. B. Stevens, Environ. Res., 142 (1987).

43. A. M. Derfus, W. C. W. Chan and S. N. Bhatia, Nano Lett., 11 (2003).

44. Y. Sun, W. Feng, P. Yang, C. Huang and F. Li, Chem. Soc. Rev., Ahead of Print (2014).

45. L. Dong, D. An, M. Gong, Y. Lu, H.-L. Gao, Y.-J. Xu and S.-H. Yu, Small, 3235 (2013).

46. X. Zhai, M. Yu, Z. Cheng, Z. Hou, P.-a. Ma, D. Yang, X. Kang, Y. Dai, D. Wang and J. Lin, Dalton Trans., 12818 (2011).

47. Y. Dai, C. Zhang, Z. Cheng, P. a. Ma, C. Li, X. Kang, D. Yang and J. Lin, Biomaterials, 2583 (2012).

48. X. Kang, D. Yang, P. a. Ma, Y. Dai, M. Shang, D. Geng, Z. Cheng and J. Lin, Langmuir, 1286 (2013).

49. Z. Xu, Y. Gao, S. Huang, P. a. Ma, J. Lin and J. Fang, Dalton Trans., 4846 (2011).

50. C. Chen, L. K. Yee, H. Gong, Y. Zhang and R. Xu, Nanoscale, 4314 (2013).

51. P. a. Ma, H. Xiao, X. Li, C. Li, Y. Dai, Z. Cheng, X. Jing and J. Lin, Adv. Mater., 4898 (2013).

52. N. J. Farrer, J. A. Woods, L. Salassa, Y. Zhao, K. S. Robinson, G. Clarkson, F. S. Mackay and P. J. Sadler, Angew. Chem. Int. Ed., 8905 (2010).

53. F. S. Mackay, J. A. Woods, P. Heringova, J. Kasparkova, A. M. Pizarro, S. A. Moggach, S. Parsons, V. Brabec and P. J. Sadler, Proc. Natl. Acad. Sci. U. S. A., 20743 (2007).

54. L. Zhou, Z. Li, Z. Liu, M. Yin, J. Ren and X. Qu, Nanoscale, 1445 (2014).

55. D. Yang, X. Kang, P. a. Ma, Y. Dai, Z. Hou, Z. Cheng, C. Li and J. Lin, Biomaterials, 1601 (2013).

56. D. Yang, Y. Dai, P. Ma, X. Kang, Z. Cheng, C. Li and J. Lin, Chem. - Eur. J., 2685 (2013).

57. Y. Yang, B. Velmurugan, X. Liu and B. Xing, Small, 2937 (2013).

58. J. Liu, W. Bu, L. Pan and J. Shi, Angew. Chem. Int. Ed., 4375 (2013).

59. Y.-H. Chien, Y.-L. Chou, S.-W. Wang, S.-T. Hung, M.-C. Liau, Y.-J. Chao, C.-H. Su and C.-S. Yeh, ACS Nano, 8516 (2013).

60. W. Yin, G. Tian, W. Ren, L. Yan, S. Jin, Z. Gu, L. Zhou, J. Li and Y. Zhao, Dalton Trans., 3861 (2014). 
61. J.-n. Liu, W. Bu, L.-m. Pan, S. Zhang, F. Chen, L. Zhou, K.-I. Zhao, W. Peng and J. Shi, Biomaterials, 7282 (2012).

62. J. Liu, J. Bu, W. Bu, S. Zhang, L. Pan, W. Fan, F. Chen, L. Zhou, W. Peng, K. Zhao, J. Du and J. Shi, Angew. Chem. Int. Ed., 4551 (2014).

63. Y.-Y. Chen, P.-A. Ma, D.-M. Yang, Y. Wu, Y.-L. Dai, C.-X. Li and J. Lin, Chem. - Asian J., 506 (2014).

64. C. Li, D. Yang, P. a. Ma, Y. Chen, Y. Wu, Z. Hou, Y. Dai, J. Zhao, C. Sui and J. Lin, Small, 4150 (2013).

65. Y. Dai, H. Xiao, J. Liu, Q. Yuan, P. a. Ma, D. Yang, C. Li, Z. Cheng, Z. Hou, P. Yang and J. Lin, J. Am. Chem. Soc., 18920 (2013).

66. D. Yang, Y. Dai, J. Liu, Y. Zhou, Y. Chen, C. Li, P. a. Ma and J. Lin, Biomaterials, 2011 (2014).

67. F. Zhang, G. B. Braun, A. Pallaoro, Y. Zhang, Y. Shi, D. Cui, M. Moskovits, D. Zhao and G. D. Stucky, Nano Lett., 61 (2012).

68. B. Dubertret, M. Calame and A. J. Libchaber, Nat. Biotechnol., 365 (2001).

69. S.-M. Lee, H. Park and K.-H. Yoo, Adv. Mater., 4049 (2010).

70. A. Topete, M. Alatorre-Meda, P. Iglesias, E. M. Villar-Alvarez, S. Barbosa, J. A. Costoya, P. Taboada and V. Mosquera, ACS Nano, 2725 (2014).

71. Y.-D. Jin and X.-H. Gao, J. Am. Chem. Soc., 17774 (2009).

72. H. Wang, Y. Wu, R. Zhao and G. Nie, Adv. Mater., 1616 (2013).

73. J. Song, J. Zhou and H. Duan, J. Am. Chem. Soc., 13458 (2012).

74. W. Wu, J. Shen, P. Banerjee and S. Zhou, Biomaterials, 7555 (2010).

75. D. N. Heo, D. H. Yang, H.-J. Moon, J. B. Lee, M. S. Bae, S. C. Lee, W. J. Lee, I.-C. Sun and I. K. Kwon, Biomaterials, 856 (2012).

76. B. Book Newell, Y. Wang and J. Irudayaraj, Eur. J. Med. Chem., 330 (2012).

77. Z. Zhang, L. Wang, J. Wang, X. Jiang, X. Li, Z. Hu, Y. Ji, X. Wu and C. Chen, Adv. Mater., 1418 (2012).

78. S. Pandey, M. Thakur, A. Mewada, D. Anjarlekar, N. Mishra and M. Sharon, J. Mater. Chem. B, 4972 (2013).

79. A. J. Cole, V. C. Yang and A. E. David, Trends Biotechnol., 323 (2011).

80. M. Das, D. Mishra, P. Dhak, S. Gupta, T. K. Maiti, A. Basak and P. Pramanik, Small, 2883 (2009).

81. S. Santra, C. Kaittanis, J. Grimm and J. M. Perez, Small, 1862 (2009).

82. J. S. Basuki, H. T. T. Duong, A. MacMillan, R. B. Erlich, L. Esser, M. C. Akerfeldt, R. M. Whan, M. Kavallaris, C. Boyer and T. P. Davis, ACS Nano, 10175 (2013).

83. W. Chen, Y. Cao, M. Liu, Q. Zhao, J. Huang, H. Zhang, Z. Deng, J. Dai, D. F. Williams and Z. Zhang, Biomaterials, 7895 (2012).

84. J. Zheng, G. Zhu, Y. Li, C. Li, M. You, T. Chen, E. Song, R. Yang and W. Tan, ACS Nano, 6545 (2013).

85. C. Li, T. Chen, I. Ocsoy, G. Zhu, E. Yasun, M. You, C. Wu, J. Zheng, E. Song, C. Z. Huang and W. Tan, Adv. Funct. Mater., 1772 (2014).

86. G. Pratx, C. M. Carpenter, C. Sun, R. P. Rao and L. Xing, Opt. Lett., 3345 (2010).

87. G. Pratx, C. M. Carpenter, C. Sun and L. Xing, IEEE Trans. Med. Imaging, 1992 (2010).

88. C. M. Carpenter, G. Pratx, C. Sun and L. Xing, Phys. Med. Biol., 3487 (2011).

89. H. Chen, T. Moore, B. Qi, D. C. Colvin, E. K. Jelen, D. A. Hitchcock, J. He, O. T. Mefford, J. C. Gore, F. Alexis and J. N. Anker, ACS Nano, 1178 (2013).

90. H. Chen, B. Qi, T. Moore, F. Wang, D. C. Colvin, L. D. Sanjeewa, J. C. Gore, S.-J. Hwu, O. T. Mefford, F. Alexis and J. N. Anker, Small, 3364 (2014).

91. Y. Sun, W. Cao, S. Li, S. Jin, K. Hu, L. Hu, Y. Huang, X. Gao, Y. Wu and X.-J. Liang, Sci. Rep., 3036 (2013).

92. S. N. Baker and G. A. Baker, Angew. Chem. Int. Ed., 6726 (2010).

93. M. Zheng, S. Liu, J. Li, D. Qu, H. Zhao, X. Guan, X. Hu, Z. Xie, X. Jing and Z. Sun, Adv. Mater., 3554 (2014). 